Revue

Sciences / LetTres
Revue Sciences/Lettres

2| 2014

Épistémologies digitales des sciences humaines et sociales

\title{
Le Liberlabo de PSL
}

Des bibliothèques-laboratoires sur le campus Paris Sciences et Lettres

\author{
Laure Léveillé
}

\section{(2) OpenEdition}

Journals

Édition électronique

URL : http://journals.openedition.org/rsl/486

DOI : $10.4000 /$ rsl.486

ISSN : 2271-6246

Éditeur

Éditions Rue d'Ulm

\section{Référence électronique}

Laure Léveillé, «Le Liberlabo de PSL », Revue Sciences/Lettres [En ligne], 2 | 2014, mis en ligne le 07 octobre 2013, consulté le 20 avril 2019. URL : http://journals.openedition.org/rsl/486 ; DOI : 10.4000/ rsl.486

Ce document a été généré automatiquement le 20 avril 2019.

(c) Revue Sciences/Lettres 


\section{Le Liberlabo de PSL}

Des bibliothèques-laboratoires sur le campus Paris Sciences et Lettres

\section{Laure Léveillé}

Remarque liminaire

:le présent article témoigne de la genèse du projet documentaire commun aux bibliothèques de la

Fondation PSL et de son état au début de l'année 2012. Si le Liberlabo n'est plus d'actualité dans tous ses aspects, il a paru toutefois intéressant de partager avec les publics, étudiants, chercheurs et enseignants-chercheurs, ces réflexions sur l'outil en pleine transformation qu'est la bibliothèque de recherche.

Évoqué pour la première fois à la conférence de presse inaugurale du campus PSL, le 16

» répond alors à une commande expresse dans le cadre du Plan Campus

: imaginer, tout en promouvant la coopération documentaire souhaitée par les cinq chefs

d'établissements concernés

, les services que pourrait offrir une bibliothèque de recherche, riche de nombreux fonds pluriséculaires, à l'horizon 2020. Sous ce vocable nouveau, « Liberlabo 
", s'esquisse alors un outil d'une génération nouvelle, du moins dans le paysage académique français dont on sait, depuis l'alerte fameuse du Rapport Miquel en 1989, qu'il reste bien en retrait de ses compétiteurs étrangers quant à la place accordée aux bibliothèques sur les campus et dans la stratégie universitaire. Un quart de siècle après cette alerte, qu'il s'agisse de pouvoir politique dans les instances, de répartition des moyens ou de poids structurel dans le développement des campus, la bibliothèque de recherche française est loin d'être considérée à la mesure des enjeux scientifiques et

pédagogiques qui lui sont liés. Le modèle nord-américain, importé dans bien des domaines de la modernisation universitaire, inspire moins, semble-t-il, lorsqu'il s'agit d'investir massivement et de façon plus ambitieuse dans l'achat de livres, d'e-books et abonnements électroniques coûteux, dans la qualité des espaces dévolus aux sources et ressources du savoir ou encore dans la formation de pointe des équipes. Tout juste s'efforce-t-on d'aller vers de plus grandes amplitudes horaires d'ouverture, admirant à juste titre ces grandes bibliothèques de Harvard ou de Stanford, ouvertes jour et nuit

; mais l'effort ne saurait se limiter à ces heures gagnées si la qualité intrinsèque des collections et services offerts ne suit pas. Pire, tout en prônant l'extension des horaires à tout prix -

y compris celui d'une moindre compétence et d'une plus grande précarité des personnels d'accueil

- on s'abrite derrière le mythe du « miracle numérique

", l'adoption univoque d'usages individuels et nomades pour décréter bien vite l'inutilité croissante des bibliothèques, anticiper la récupération profitable de leurs locaux et de leurs moyens, négliger leurs besoins réels et ceux de leurs publics divers, à commencer par les étudiants et apprentis-chercheurs. Dans ce contexte, le projet Liberlabo de PSL

plaide pour l'investissement financier bien sûr, mais aussi politique, conceptuel et symbolique, que méritent les bibliothèques au sein d'un groupement scientifique ambitieux aux fonds considérables et prestigieux, tous biens publics et patrimoniaux que PSL a pour mission de préserver, enrichir et diffuser.

Articulé autour du continuumlire-écrire-prescrire-évaluer

, qui caractérise l'activité de l'enseignant-chercheur comme, bien vite, celle de leurs étudiants avancés, le Liberlabo cherche à réunir et à décloisonner

: les disciplines, les processus à l'œuvre dans le travail intellectuel, pédagogique et scientifique, les métiers au service de la recherche et de la formation par la recherche. Ce faisant, il parie sur le renouvellement et la fécondité induits par de nouvelles configurations spatiales et fonctionnelles au service des

lecteurs-auteurs

de la science, et non plus des seuls lecteurs en quête de ressources bibliographiques. Science s'entend ici au sens large puisque presque toutes les disciplines sont représentées sur le campus PSL, lequel revendique en outre sa spécificité et sa vocation pluridisciplinaires.

Le Liberlabo souhaite, comme la plupart des grandes bibliothèques aujourd'hui, offrir un éventail élargi d'assistance et de services autour de grandes collections multisupports, sur site et à distance, sans rompre avec la riche tradition des bibliothèques de recherche, dont PSL abrite quelques beaux fleurons, convoités à juste titre. 
${ }^{3}$ Depuis la rentrée 2010, le projet initial a été intégré au volet « Diffusion des savoirs » de la candidature PSL aux Initiatives d'excellence (IDEX)

; un groupe de travail formé des directeurs de ces bibliothèques partenaires - d'abord au nombre de 5, puis 12 à compter du début 2011, puis 16 - a poursuivit les travaux initiés par la phase de candidature.

Tout en y souscrivant absolument, le Liberlabo souhaite dépasser le strict cadre des coopérations déjà identifiées et nécessaires, entre bibliothèques de recherche (réseaux documentaires et/ou thématiques, catalogue commun, conservation partagée, numérisation, etc.) pour les intégrer à un équipement à la fois multifonction et cohérent, qui convoque ensemble des métiers aujourd'hui cloisonnés et dispersés (bibliothèque, documentation, archives, édition universitaire, diffusion des savoirs, systèmes

d'informations, bibliométrie, conseil juridique, etc.), au service des publics habituels comme d'une plus grande ouverture sur la cité, la diversité des usages et leur compatibilité gouvernant l'invention de nouvelles configurations spatiales. C'est là toute la dimension prospective que ce projet tente de construire en réponse à l'incitation de l'appel d'offres IDEX qui, rappelons-le, invitait à inventer des actions « structurantes et

》(sic)

. Mais c'est aussi tout le paradoxe pour un univers, celui des bibliothèques, qui déjà peine à financer des actions coopératives promues depuis plusieurs années, vite perçues comme

", alors même qu'elles sont aussi la clé de toutes les évolutions ultérieures

de « vieux projets

: ne citons que les opérations de conversion rétrospective des fichiers papier, ou « rétroconversion

", qui par leur nom même ont injustement l'air de regarder davantage dans le rétroviseur que de proposer une vision des bibliothèques de demain. Ce sont elles pourtant qui nourrissent la vaste bibliothèque numérique universelle que de grands groupes privés prétendent aujourd'hui bâtir, plus vite que la puissance publique.

Aussi peut-on définir brièvement le Liberlabo par cette formule :

davantage qu'une bibliothèque autour d'une bibliothèque

. En cela, le nouveau nom proposé ne signifie pas que celui de bibliothèque est obsolète, il veut s'y associer, non s'y substituer, pour signifier la vitalité d'un métier de pointe au cœur de la recherche et de l'IST

. À l'heure où de grandes sociétés internationales se sont emparées de ce champ on ne peut plus fertile, celui des contenus livresques et de leurs métadonnées, le métier de bibliothécaire est, en coulisses, un des plus porteurs et des plus rentables - en coulisses seulement, car le discours en vogue est plutôt à l'affirmation que les bibliothèques sont dépassées, étrange aveuglement quant à la captation, à des fins économiques, du métier dont les nouvelles technologies de la recherche ont précisément le plus besoin.

\section{Bibliothécaire ou le métier prospère des métadonnées}

Là où les grands éditeurs privés scientifiques font payer plusieurs fois aux chercheurs

in extenso, Google, sans grande concurrence à ce jour, fournit « gratuitement 
" aux grandes bibliothèques nationales et académiques qui ont contracté avec lui, les textes intégraux numérisés en masse dans de mystérieuses implantations techniques, en échange de métadonnées descriptives, intégralement élaborées et livrées par les bibliothécaires, moyennant des heures d'un travail rigoureux et exhaustif de normalisation (notices en format MARC, catalogage particulièrement fouillé et structuré pour les ouvrages académiques pointus en langues diverses et rares). Or ce travail technique de très longue haleine -

il faut en moyenne vingt minutes pour cataloguer, ouvrage en main, un document scientifique de haut niveau et permettre ainsi aux requêtes en ligne toute la pertinence voulue en réponse

- est rémunéré par la puissance publique, comme l'est celui des chercheurs qui écrivent les contenus. À ce jour, un document né numérique n'exige pas moins de temps de travail humain qu'un écrit papier, livre ou article de revue, qu'il s'agisse de l'écrire, de l'éditer ou d'en décrire les métadonnées de façon intelligente et rigoureuse, processus complexe qui ne saurait être entièrement automatisé.

De même que le mouvement des archives ouvertes, initié par les chercheurs, invite la communauté de recherche à se réapproprier les contenus qu'elle produit et leur libre circulation, de même les bibliothèques, en coopération étroite avec les chercheurs,

: sans elles, sans leur savoir faire de description normalisée aux formats internationaux, pas de mise en réseau efficace des données, pas d'échanges internationaux entre

catalogues de référence, et surtout, beaucoup de bruit ou de silence sur le web. On sait déjà qu'un livre mal rangé sur les rayons est un livre perdu ; il en est de même dans la grande bibliothèque universelle que pourrait devenir le web. Un document mal décrit est un document perdu

, vers lequel aucune requête, si précise soit-elle, ne pointera jamais. Une donnée mal indexée ou approximativement signalée est une donnée qui, virtuellement, n'existe pas

. Google l'a bien compris qui compte précisément sur le travail gigantesque, sédimenté sur plusieurs siècles, accompli par les équipes successives de bibliothécaires dans toutes les grandes institutions de lecture scientifiques et académiques, de la Bodléian d'Oxford à la Bibliothèque cantonale universitaire de Lausanne, en passant par la bibliothèque universitaire de Gand ou la bibliothèque nationale de Bavière, pour ne citer que les grandes européennes qui ont signé les premières avec le « géant ».

Nombre de chercheurs disent en toute franchise qu'ils ne mettent plus les pieds à la bibliothèque alors qu'ils s'y rendent sans cesse du bout des yeux, à leur insu parfois, virtuellement ou plutôt à distance, pour consulter des ressources auxquelles ils n'auraient pas accès sans le budget de la bibliothèque, le service « acquisition

» de la bibliothèque, le traitement des métadonnées et le signalement par l'équipe « catalogage 
» de la bibliothèque, etc. Une telle méconnaissance mériterait quelques actions pédagogiques au risque d'incommoder les lecteurs pressés de ressources électroniques

: une alerte rapide rappelant « Vous accédez à cet article grâce à la bibliothèque qui a dépensé tant d'euros et employé tant d'ETP pour ce service ». Peut-être les budgets documentaires seraient-il alors plus aisés à négocier dans les instances où siègent les mêmes chercheurs.

\section{Initiatives de chercheurs, réponses de bibliothèques, pour un espace de réappropriation des outils}

De pionnières initiatives, telles que celle du Cléo - Centre pour l'édition électronique ouverte - connu d'abord pour son site de périodiques en ligne, Revues.org, puis désormais pour sa plate-forme collaborative de carnets de recherche, Hypothèses.org, et son agenda scientifique partagé, Calenda, invitent depuis quelque temps les bibliothèques à rejoindre

le mouvement de l'édition et de la science ouvertes. Ces dernières n'ont pas encore

vraiment répondu à l'appel, bridées qu'elles sont par les impératifs budgétaires

: le Cléo a bien diagnostiqué le problème majeur posé par la hausse exponentielle du coût des abonnements électroniques aux journaux scientifiques. Ces abonnements, qui peuvent croître de 15

\% par année, grèvent littéralement le budget des institutions, aux dépens d'autres acquisitions et de l'évolution des services. La demande perpétuelle de justification des dépenses, le temps englouti dans des négociations difficiles

- à chaque éditeur son modèle économique, sans compter le déséquilibre patent des forces en présence pour de tels enjeux financiers - grèvent le temps de travail des bibliothécaires, qui, de ce fait, en viennent à négliger parfois la mise en forme de l'offre numérique gratuite.

Peu de portails documentaires valorisent à leur juste place les ressources des archives et de l'édition ouvertes, ceci davantage en SHS qu'en sciences dites dures,

; certains proposent des listes de signets mais, faute d'outils appropriés, peinent à y consacrer le temps de veille et mise à jour des adresses et des métadonnées d'accès. De ce fait, les bibliothèques sont contraintes de négliger leurs meilleurs alliés, et surtout leurs partenaires naturels au sein des communautés de recherche. Le Liberlabo, dans son nom même, souhaite réaffirmer cette alliance et cette communauté d'intérêts profonds

:liber

, pour la liberté et la gratuité du libre accès aux contenus pas ceux-là même qui les créent ; pour l'organisation, en collaboration avec les bibliothécaires, de l'accès le plus fluide, le plus pertinent et le moins coûteux possible à ces contenus (reste toujours, irréductible, le temps de travail qui fournit la valeur ajoutée du document) ;labo 
, parce que ce travail de mise en forme et de mise en valeur des contenus académiques ne peut se faire sans un lieu, à la fois physique et virtuel, de convergence des compétences à

l'œuvre et de réflexivité sur les pratiques. Dans ce lieu, ou à partir de lui, pourra être observée cette nouvelle boucle du "read-write»

- toute cette vie des communautés autour du texte, qui en assurent la diffusion et les métamorphoses au fil des recommandations et autres gloses en ligne - cette culture nouvelle du "remix »évoquée par Lawrence Lessig, le juriste à l'origine des licencescreative commons.?

En effet, de même que la notion d'œuvre littéraire et celle d'auteur se voient métamorphosées, pour ne pas dire désintégrées parfois, par le "read-write»

, de même les publications scientifiques peuvent et vont sans nul doute voir éclater et évoluer leur forme, en tout cas coexister des formes anciennes, finies, avec de nouvelles, réinscriptibles à l'infini. Il serait grave que les auteurs scientifiques, en l'occurrence nos publics des bibliothèques académiques, désertent cette opération réflexive sur leurs pratiques d'écriture et de lecture. Le Liberlabo s'offre à eux comme un des laboratoires possibles de ce chantier gigantesque, à peine ouvert et formidable. Rejoignant l'analyse de l'historien du livre et grand directeur de la Bibliothèque de Harvard, Robert Darnton, dans son dernier essai

L'Apologie du livre

, le Liberlabo, par sa double déclinaison, physique et virtuelle, de services sur place et en ligne, signifie que la bibliothèque comme espace et le livre comme objet ne sont pas dépassés mais indispensables en termes de préservation des sources et ressources de la science. Il postule toutefois qu'ils peuvent être revivifiés, plutôt que menacés, par la révolution numérique qui elle-même s'en nourrit. Le Liberlabo ne renie pas la bibliothèque - ce n'est pas là le sens de son avènement comme nouveau mot - mais affirme qu'elle peut et doit être au cœur d'un processus nouveau, pour accompagner les chercheurs, étudiants-chercheurs et enseignants-chercheurs dans les mutations morphologiques de l'écriture et de la lecture scientifiques qu'induit la révolution numérique. Or cet accompagnement ne sera pertinent que s'il prend en compte le continuum diachronique et synchronique des supports, qu'il s'agisse d'un manuscrit, d'un livre ancien relié en parchemin, d'une vidéo ou d'une bande numérique, pour y appliquer selon des savoir-faire différents mais avec la même rigueur, les missions fondamentales des bibliothèques

: collecter, sauvegarder et communiquer, diffuser. En ce sens, le Liberlabo voudrait offrir, pour la totalité des collections multisupport, un espace physique de travail et d'échange aux types de processus que les plates-formes collaboratives telles que celle du Cléo (Revues.org, Hypothèses) proposent en ligne pour les productions scientifiques, tout en promouvant leur observation et analyse. 


\section{Des bibliothèques-laboratoires pour des lecteurs- auteurs}

8

Le Liberlabo est né du constat simple que les usagers des bibliothèques d'enseignement supérieur et de recherche sont des auteurs autant que des lecteurs

; des auteurs en herbe pour les étudiants rédacteurs d'un premier mémoire, des auteurs de plus en plus confirmés par la suite, des « publiants

» comme dit le jargon nouveau de l'évaluation. Ces lecteurs-auteurs sont aussi des prescripteurs, aussi bien à l'égard des bibliothécaires auxquels ils suggèrent les achats pertinents et, parfois, les classifications idoines - ce qui est encore plus flagrant dans les

bibliothèques très spécialisées

- qu'auprès de leurs étudiants auxquels ils recommandent les lectures incontournables. Ils sont en outre des évaluateurs, de leurs étudiants, de leurs collaborateurs et de leurs pairs. Le Liberlabo intègre ce continuum de pratiques à l'évolution des relations entre enseignants-chercheurs et apprentis-chercheurs

: à ce titre, s'il faut, comme on y enjoint les candidats aux divers appels d'offres, le situer dans l'offre nationale et internationale de services comparables, le Liberlabo se situe d'une certaine manière dans le prolongement des learning centres

déjà bien connus en Amérique du Nord et dans certains pays d'Europe (citons le Rolex Learning centre de l'EPFL en Suisse ou celui de l'université de Glasgow)

; mais il s'adapte ici au contexte spécifique des bibliothèques et publics de PSL. Moins destinés aux premiers cycles universitaires qu'à celui de la formation à la recherche par la recherche ainsi qu'aux besoins de la recherche avancée, le Liberlabo serait davantage un "searching and writing center"

. Il a paru souhaitable et pertinent de le distinguer encore par un nom qui s'affranchît de l'anglo-saxon, même si la candidature aux IDEX devait être rédigée en anglais.

Encadré 1 - « Liberlabo » : un nouveau mot pour un nouvel espace au service de la recherche

« Liberlabo

» désigne une offre de service structurée autour du réseau documentaire mais excédant les missions traditionnelles des bibliothèques. C'est un vocable potentiellement universel sans être anglo-saxon

: la sémantique multiple de « liber 
» évoque à la fois les libertés et fluidité d'accès aux services grâce à la révolution numérique mais aussi le libre accès aux rayons, pionnier dans les bibliothèques de PSL, les problématiques de l'open-access pour les contenus scientifiques, l'ouverture sur la cité, et le continuum fécond avec le substrat patrimonial pluriséculaire de PSL, rendu par l'hommage au latin des humanistes. Le choix de « liber

" signifie, par-delà les mutations morphologiques de la production écrite, l'ancrage des pratiques actuelles dans les sources et grandes filiations scientifiques, la recherche en train de se faire ayant demain vocation à s'inscrire à son tour dans ce patrimoine de réputation internationale. Le Liberlabo réaffirme en le développant le rôle vivant et vital de laboratoire que jouent les bibliothèques en sciences humaines

et sociales

; le projet postule que les sciences, à l'instar des mathématiques, peuvent y trouver un intérêt renouvelé dans le croisement fécond des disciplines et des méthodes, comme dans la valorisation de leur patrimoine, souvent inexploité et méconnu.

Dépassant les vaines oppositions entre papier et électronique, patrimoine et nouvelles technologies, il les réunit au service de la recherche, de ses évolutions propres comme de sa réflexion épistémologique. Le Liberlabo peut devenir l'un des observatoires privilégiés de la vie des disciplines, lieu possible de leur émergence et de leurs croisements, un des acteurs et témoins de leur histoire comme de leur genèse.

\section{Ce nouvel outil est d'abord un espace}

: il ne s'agit pas de bâtir un nouveau lieu unique où fusionneraient collections, équipes et services, mais de créer un espace physique, matérialisé par des parcours topographiques inscrits dans le campus et la cité

, entre des bibliothèques, une quarantaine, pour la plupart historiques, dont certaines sont déjà porteuses d'une ambition architecturale en centre-capitale. Un tel espace a vocation à réunir davantage les générations de chercheurs autour d'outils communs, de plates-formes collaboratives et d'espaces d'échanges, destinés à la lecture

et

à l'écriture scientifiques. Il prolonge ces outils communautaires, façonnés à l'écoute de ces usagers premiers, par des outils de diffusion des savoirs à l'attention de tous les publics potentiels que motive la science en train de se faire. Cette dernière mission est évidemment facilitée par le versant virtuel du Liberlabo, ou « mon Liberlabo virtuel

» devient aussi un espace, numérique cette fois, de dialogue et service à distance, personnalisé à la demande, apte à répondre aux besoins diversifiés de différents profils

d'utilisateurs. L'une des démarches professionnelles du Liberlabo consiste à imaginer, chaque fois que cela est possible et opportun, le versant virtuel d'un service sur place et vice versa

. En cela, il rejoint bien sûr toute la problématique actuelle de la bibliothèque « hybride 
». On n'est pas dans une logique de substitution (de l'électronique au papier, de l'automate à la personne de service, etc.) ni dans celle d'une juxtaposition de couches technologiques successives, qui serait en cela trop esclave du rythme de production des nouvelles technologies, mais bien dans l'élaboration d'un service complet, pensé à partir de la notion de service, qui cherchent ensuite les meilleurs outils, pour sa version nomade comme pour sa version plus sédentaire - nous préférons « ancrée » dans le campus.

On l'aura compris, le Liberlabo est tout sauf un projet « clé en mains »

: il se contente à ce stade de proposer des pistes, des orientations pour inventer un nouvel outil. Pensé au service des missions et des publics qui font la spécificité de PSL, à savoir les acteurs de la formation à la recherche par la recherche, qu'il soit apprentis ou tuteurs, le Liberlabo, encore très abstrait à ce jour, serait conçu avec ce public chercheur, d'après une enquête qualitative poussée, nourrie d'un dialogue constant. Pourquoi ne pas imaginer, par exemple, des fonctionnalités et des configurations spatiales encore mieux adaptées au déploiement du tutorat, y compris un tutorat documentaire privilégié, dans un groupement d'institutions que caractérisent de longue date un taux d'encadrement exceptionnel et la qualité des relations pédagogiques possibles autour de la recherche.

\section{Encadré 2 - Missions et fonctionnalités du Liberlabo}

Du côté des usagers :

L'adoption du numérique par les chercheurs concerne toutes les phases et dimensions de leur activité, et non pas seulement l'accès aux livres ou aux journaux électroniques. Or tous ne sont pas, loin s'en faut, au même degré d'appropriation des nouvelles technologies. Le Liberlabo serait un outil et un support pour

- produire des contenus

: rédiger, déposer des travaux académiques, diffuser des cours, publier/éditer à divers degrés de validation et diverses échelles

\section{- évaluer, valider des contenus}

: recenser, rédiger des comptes rendus, valider des degrés de publication, participer à des comités de rédaction et/ou d'évaluation (revues, monographie, édition en ligne, etc.)

- sélectionner, prescrire des contenus :

bâtir des bibliographies ou des outils bibliographiques, prescrire des achats documentaires, diffuser des orientations bibliographiques (aux étudiants, aux collègues, aux bibliothécaires, aux internautes, etc.), échanger sur des lectures partagées, etc.

; 
- déposer, publier, diffuser des contenus :

individuellement ou sur des plates-formes collaboratives, avec ou sans assistance, selon degré d'autonomie divers, dans un cadre institutionnel, dans l'édition privée ou publique, au sein des archives ouvertes, sur un site ou un canal de diffusion des savoirs dans la maîtrise de la réglementation sur le droit d'auteur et la propriété intellectuelle, en toute connaissance des publics visés, etc.

\section{Du côté des professionnels :}

Certaines des missions déclinées ci-après sont des missions désormais classiques des bibliothèques

; d'autres ressortissent à ce jour des centres de ressources informatiques, services de Diffusion des savoirs, ou presses et éditions institutionnels. Cette mixité fonctionnelle fait en partie écho à celle des learning centres mais elle est davantage tournée vers le niveau ou l'activité « Recherche

» que vers les services aux étudiants de premier cycle. Le Liberlabo proposerait ensemble

:

-uneassistance 24/24 (sur place ou en ligne) à la recherche documentaire dans les collections du campus et hors les murs ;tutorat documentaire en collaboration avec le tutorat pédagogique et scientifique ;

-uneassistance informatique pour l'usage de logiciels et applications spécialisés

(ENT, outils individuels de bibliographie, prise de notes, etc. mais aussi logiciels de cartographie, archéologie, musicologie, etc.)

; assistance au développement d'applications nouvelles ;

-uneaide à l'édition numériquescientifique (au sens large, SHS incluses) ;

-uneassistance à la création/rédaction de revues pluridisciplinaires

, ainsi pour la présente revue par exemple;

-uneoffre de formation continuedans les métiers supports de la science ;

-unecouverture optimale de la bibliographie

dans les disciplines du campus (tous supports);

-lamaintenance d'une plate-forme collaborative et interactive (chercheurs et bibliothécaires)pour la constitution des collections tous supports , c'est-à-dire de fonds cohérents avec les missions du campus, cohérents avec la politique documentaire des autres partenaires du Quartier latin (rappelons quelques voisins documentaires prestigieux et précieux, telles les bibliothèques SainteGeneviève, de la Sorbonne, Cujas et la récente Sainte-Barbe) ; 


\section{-undépôt institutionnel}

et une plate-forme collaborative pour la collecte, la validation, la mise en ligne et

l'indexation pertinente, aux normes internationales, de contenus scientifiques, en collaboration directe,

in situ, avec les professionnels de la Diffusion des savoirs.

\section{Encadré 3 - Pour la mise en valeur d'un patrimoine vivant}

La « boucle

» patrimoniale ou les cycles de traitement des données courantes destinées à devenir patrimoniales (et non d'emblée patrimoniales comme le sont certaines des acquisitions auprès des libraires anciens par exemple) sont métamorphosés par le concept de « diffusion continue

» où les missions de conservation et de diffusion ne sont plus séparées. Selon le truisme « la science en train de se faire

» sera demain le précieux témoignage de la science accomplie ou de « la science telle qu'elle se faisait

", la valorisation du patrimoine scientifique au sens large est consubstantielle au concept du Liberlabo, d'où les missions suivantes

Mission de préservation de pointe, y compris l'archivage pérenne pour les contenus et supports numériques, grâce à un laboratoire de conservation moderne et une réflexion sur les pratiques mêmes de conservation (PSL offre en son sein des compétences de haut niveau aussi variées que chimie, biologie, informatique, histoire du livre, bibliothéconomie, etc.).

-Valorisation du patrimoine savant au Quartier latin pouvant s'appuyer sur :

des gisements d'archives et des sources trop peu exploités, voire méconnus car mal identifiés et non inventoriés, d'où aussi mal diffusés (papiers privés, photographies, objets et instrumentation scientifiques, estampes et dessins, etc.) ; des gisements uniques, précieux, mais souvent menacés par les impératifs des labos, eux-mêmes soumis à des contraintes spatiales et de calendrier (restructurations indispensables mais ne prévoyant pas l'espace de la préservation patrimoniale);

un silo commun de conservation/dépôt des collections moins demandées, couplé à un service de navette pour rapatriement des documents demandés au Liberlabo (sur

le modèle de celui du CTLES - Centre technique du livre de l'enseignement supérieur - à moins qu'on ne puisse compter sur l'extension rapide des capacités d'accueil de ce dernier, service public national déjà très sollicité par les grandes bibliothèques intra-muros) ; 
une enquête historique et sociologique, déjà bien entreprise, sur les parcours savants, au sens d'itinéraires intellectuels et parcours académiques, institutionnels

(prosopographie) autant que sur la mise en œuvre de parcours physiques, topographiques, sur la fameuse « montagne de livres » qu'est la Montagne Sainte-

Geneviève ;

•unemise

en perspective de cette histoire avec la valorisation du monde actuel des laboratoires, en collaboration avec toutes les initiatives en direction du primaire, $\mathrm{du}$ secondaire, du grand public, telles que la « Main à la Pâte », " Parcours des Sciences ", " Paris-Montagne », au moyen de visites, journées portes ouvertes, expériences filmées, etc. mais aussi selon des dispositifs plus périodiques sinon permanents, via une muséographie moderne et vivante au sein d'un espace « Faberlabo

$»$,

nourrie du partenariat récent avec les écoles d'art et des recherches menées dans le cadre du SACRe (problématique Art et Science).

Sans prétendre rivaliser avec des équipes de recherche, le Liberlabo, par ses sources et ressources, pourrait sans nul doute

\section{éclairer l'histoire des parcours savants et la constitution du « corps » savant}

, en invitant à regarder encore davantage les chemins physiquement et concrètement

parcourus

, les bibliothèques arpentées, les lieux et les cotes fréquentés, les postures physiques adoptées ou imposées (assis en bibliothèque par exemple) par les lieux et les outils de la science. En collaboration avec les usagers chercheurs, l'invention d'un nouvel espaceoutil, le Liberlabo, serait adossée à ce type d'enquête.

\section{Genèse du projet \\ : service public de lecture, espace-temps de la recherche et corps savant}

Né au printemps 2010, le projet Liberlabo rencontre les circonstances et se plie aux attendus d'opportunité que dictent la nouvelle donne universitaire et ses logiques

: c'est un appel à projets inspiré de la concurrence privée qui suscite l'expression, sans aucune censure, d'une prospective professionnelle à des niveaux de financement inédits, alors même que le Liberlabo s'inspire d'un goût profond et d'un souci constant du service public.

L'affirmation du Liberlabo comme lieu, espace physique incontournable et non dépassé de la recherche, vient tout droit d'une thèse consacrée à la genèse du service public de 
Sous la Troisième République, dans le prolongement de l'école primaire et parfois dans

la bibliothèque publique moderne naît d'abord de la conquête d'un espace propre

ses murs,

- de l'armoire au fond d'un préau en passant par l'annexe d'une mairie jusqu'à

l'inauguration d'un espace « modèle

", ouvert et agréable, inscrit dans la cité, souvent inspiré des réalisations nordaméricaines et des conseils pratiques prodigués par les femmes bibliothécaires venues enseigner leur métier à Paris après la Première Guerre mondiale. La bibliothèque de

L'Heure joyeuse fut un de ces laboratoires d'une lecture plus ouverte et attrayante, faisant bonne part aux couleurs et à la gaieté, parce que d'abord destinée aux enfants et au loisir éducatif. Tandis qu'elle s'affranchit peu à peu de la tutelle de l'école et du seul souci d'encadrement postscolaire, la bibliothèque publique naissante, ouverte à tous, conciliant l'étude et le divertissement, gagne sa place aux côtés des autres services de la

cité moderne, inscrite dans l'espace municipal notamment, sans toutefois parvenir à égaler l'inscription dans la vie quotidienne du citadin dont témoignent, entre autres, le cinéma et les séries télévisées du domaine nord-américain. Plus récemment, le renouveau

de la lecture publique française à partir des années 1980 se lit principalement dans

l'espace urbain des métropoles et capitales régionales, avec des réalisations architecturales qui cherchent à manifester l'ouverture, la transparence, l'accessibilité et

la diversité des supports et des services. Par-delà les usages premiers de la lecture, la médiathèque municipale est bien souvent le seul espace public ouvert gratuitement aux heures de loisir, le soir et le week-end, rare alternative au jardin public lorsqu'il fait gris ou froid. Comme les cafés, elle offre un lieu où l'on peut lire - travailler ou se distraire -

au milieu ou à côté des autres sans être nécessairement avec ces autres. À l'inverse du

café, elle est silencieuse (théoriquement et principalement) et n'exige aucune consommation payante. On ne lui trouve donc guère d'équivalent dans l'espace public, ce qui plaide suffisamment pour sa sauvegarde et son développement. Présentant son récent

\section{La Société des égaux}

ouvrage,

, Pierre Rosanvallon, titulaire de la chaire d'histoire moderne et contemporaine du politique au Collège de France, constate et déplore qu'il n'y a plus guère de lieu dans la cité dite démocratique, où pauvres et riches se rencontrent et se côtoient

. La médiathèque moderne est peut-être un de ces rares lieux de rencontre des générations et des classes sociales, comme en témoigne, entre autres réalisations récentes, la bibliothèque de l'Alcazar à Marseille.

Aussi y aurait-il quelque intérêt, pour imaginer les bibliothèques de recherche du futur, à 20 voir aussi du côté des

ideas stores

dans des contextes nationaux où la configuration administrative n'a pas autant cloisonné le monde des bibliothèques publiques, territoriales et celui des universitaires et académiques. Il existe en France une partition entre deux grandes tutelles,

l'Enseignement supérieur-Recherche d'une part et la Culture d'autre part, partition qui traverse d'ailleurs le paysage documentaire de PSL puisque les bibliothèques des écoles d'art dépendent pour partie du ministère de la Culture. Or il semble que les bibliothèques territoriales, au travers des grandes réalisations de ces trois dernières décennies, aient davantage pensé la question de l'espace bibliothèque dans la cité. 
Alors que le réfectoire des Cordeliers, à l'Université Paris-Descartes, venait d'accueillir, en

", l'envie était grande de réfléchir au corps immobile (du moins en apparence), au corps sédentaire (du moins le plus souvent), qu'est le corps du savant au travail, lorsqu'il ne parcourt pas le monde, en pérégrinations académiques (du moins dans sa version

occidentale au

XXI

siècle). Les mutations technologiques rendent certes les voyages moins handicapants - on emporte son biotope technologico-académique et on se connecte presque partout - mais n'améliorent guère les postures si l'on en croit les statistiques des maladies professionnelles et autres TMS (troubles musculo-squelettiques) directement liés au travail sur écran. Explorons peut-être davantage les aspects du corps contraint, celui auquel les bibliothèques, les amphithéâtres, les salles de cours et les bureaux de chercheur (lorsqu'ils existent) n'offrent guère qu'une ou deux postures, tolérées et possibles, à savoir assis, sur un siège plus ou moins mobile, à une table plus ou moins large et haute. Le Liberlabo postule que la bibliothèque a une responsabilité et un rôle nouveau à jouer dans l'organisation et la souplesse possibles de l'espace-temps du chercheur. Sans doute constitue-t-elle d'ailleurs un pivot singulier de l'articulation de l'espace et du temps, ne serait-ce que dans l'organisation spatiale des cotes, classifications thématico-temporelles en grande part, qui régissent les parcours de l'usager dans les sous-espaces visibles. La temporalité accélérée qui s'impose à l'activité de recherche comme à tant d'autres - bien souvent à tort hélas - exige au moins qu'on se penche sur la fluidité croissante des services et leur contiguïté/proximité efficace au service des chercheurs qui ne peuvent plus se disperser ni gaspiller le temps précieux de l'analyse, de l'expérience et de l'écriture. Là encore il s'agit de faire en sorte que le gain de temps ne se fasse pas au détriment du collectif, des rencontres et des échanges autres que virtuels. Inventer le Liberlabo c'est imaginer un nouvel espace-temps du travail de recherche dans

la relation et dans l'espace collectif public tout en accordant sa pleine place au corps individuel du chercheur.

\section{Encadré 4}

Venez découvrir et tester le scriptorium, bureau modulable imaginé pour les chercheurs par deux jeunes designers, Lucas Hoffstalt et Samuel Lamidey, dans le cadre de leur fin de scolarité à l'Ensad en 2009. Ce meuble est à la fois visible et utilisable par les lecteurs à la Bibliothèque générale du Collège de France depuis le 23

janvier 2012. Vos réactions et remarques seront utiles à l'enquête Liberlabo sur la diversification souhaitable des espaces et des postures de travail. Attention : le scriptorium sera bientôt rendu à ses concepteurs (automne 2013). 


\section{Revitaliser les territoires de la science : réappropriation des sources, invention des outils}

Le Liberlabo est, de fait, un projet militant ; il l'est dès son origine

; tandis que les évolutions récentes du projet collectif sont assumées par tout le groupe des directeurs de bibliothèques impliqués, l'auteur du présent article n'engage qu'ellemême dans ce propos plus politique. Son statut atypique de maître de conférences reconvertie dans les bibliothèques sans avoir le titre de conservateur, ses expériences successives de lectrice étudiante, puis doctorante, puis d'enseignant-chercheur, puis de directrice de plusieurs bibliothèques de recherche, lui inspirent ensemble cette proposition d'évolution institutionnelle. Le Liberlabo milite d'abord pour la juste place des bibliothèques de recherche dans la politique scientifique, au sein des territoires de la science et avec tous les acteurs de la science

; en cela il entérine la position de la majorité des directeurs de bibliothèque comme de leur tutelle professionnelle. Il lutte contre la tendance dommageable à considérer ce « service support

» comme un parmi d'autres services centraux traditionnellement dirigés par un cadre supérieur administratif. Il est en complète cohérence avec le décret du 23 août 2011, qui réaffirme la légitimité d'une tutelle présidentielle et scientifique directe sur les directeurs de bibliothèques d'enseignement supérieur et de recherche. Que des directions de bibliothèques, responsables de grosses équipes et de gros budgets, collaborent avec des directeurs généraux des services compétents en matière de bonne gestion, cela est une nécessité

; qu'ils puissent justifier du meilleur usage des deniers publics, c'est une juste requête ; mais qu'une tutelle purement administrative fasse écran entre ces directions, de statut scientifique ou assimilé, et la présidence scientifique des établissements, cela n'est pas approprié aux missions fondamentalement scientifiques des bibliothèques de recherche.

Aussi le Liberlabo s'offre-t-il comme espace de relance et de déploiement du dialogue entre apprentis-chercheurs, chercheurs et bibliothécaires, y compris au plus haut niveau des stratégies scientifiques et documentaires. C'est avant tout pour les bénéfices de la recherche et des chercheurs que les bibliothécaires défendent cette juste place dans les nouveaux organigrammes de l'université autonome

: aussi espèrent-ils trouver dans la communauté de recherche un allié naturel dans l'éclosion d'une nouvelle politique des bibliothèques de recherche, revitalisée, qui contribue à la vitalité de la recherche.

Dans cette entreprise, il faut bien sûr s'attendre à quelques résistances, dont certaines se sont déjà manifestées dans la phase de candidature. Ne nous cachons pas les enjeux politiques, économiques et stratégiques sous-jacents 
: que les communautés scientifiques se donnent les moyens, dans la suite de toutes les initiatives type OAI - Open Archives Initiative -, de s'affranchir vraiment des modèles économiques qu'on leur impose (captivité de grands éditeurs privés, coûts exponentiels des abonnements, garantie faible ou nulle sur la pérennité des accès aux archives, etc. - toutes problématiques désormais bien connues des chercheurs comme des bibliothécaires) en coopérant pour inventer et promouvoir de nouveaux modèles. À cet égard, l'action d'une unité mixte de service telle que le Cléo sensibilise les communautés de recherche et la société tout entière à la problématique des biens communs de la connaissance

; il en va de la réappropriation des savoirs par leurs acteurs et par la société civile comme de celle des semences et de la production agro-alimentaire. Les nourritures intellectuelles sont menacées de verrouillage comme le sont déjà les nourritures terrestres et les conservatoires de graines et semences, devenus illégaux et militants, ressemblent étrangement à des bibliothèques.

Aider les chercheurs à se réapproprier les sources, les données, les contenus, les conditions de leur diffusion, leur évaluation à la fois qualitative et quantitative, tous les outils de la science, valoriser pour eux et avec eux les espaces-outils communautaires, font partie des ambitions du Liberlabo comme lieu d'expérimentation d'un autre espacetemps de la recherche en tant que portée par des communautés et non par une juxtaposition d'individus seulement en compétition, engagés dans la course à la publication. À terme, pourquoi le Liberlabo ne serait-il pas en outre un des lieux privilégiés de fabrication, collecte, indexation et sauvegarde des indicateurs les plus pertinents de la science

? des indicateurs quantitatifs et qualitatifs, toujours fondés sur une observation vivante des pratiques, des indicateurs évolutifs, construits en concertation constante avec tous

les acteurs. Dans la mesure où l'unité qu'est la publication joue un tel rôle dans l'évaluation de la science et dans sa diffusion, le Liberlabo deviendrait un outil de valeur ajoutée à l'excellence du chercheur dans cet acte fondamental d'écrire et de publier. Le lieu de croisement des personnes, des disciplines et des compétences, toutes adossées à des outils de pointe ne deviendrait-il pas le meilleur observatoire des pratiques institutionnelles, de leurs évolutions, de leurs apports à la communauté

? Au risque d'être à la fois juge et partie répond l'avantage en tout cas de l'autoévaluation nuancée et de la contribution à faire évoluer des outils performants et intégrés au service de la science.

Parmi les résistances possibles il ne faut pas sous-estimer les corporatismes et la crainte des métiers d'être dépossédés ou de voir les territoires et frontières professionnels

brouillés

: le patrimonial et le documentaire entre autres alors qu'il y a pourtant un enjeu scientifique majeur à pouvoir pointer du texte académique vers ses sources, que de pouvoir relier autant que possible la donnée de pointe à la sédimentation patrimoniale qui fonde souvent son éclosion ou qui la prépare. Il y a aussi le manque de curiosité pour les apports de métiers moins souvent associés à la recherche 
: psychomotriciens, ergonomes, architectes, designers, psychanalystes, scénographes, pourraient sans doute éclairer d'un autre jour la coopération des chercheurs, des bibliothécaires, des informaticiens, des archivistes et autres métiers impliqués initialement dans un projet de coopération des bibliothèques. Il est difficile de vaincre pêle-mêle la peur - le tabou

? - l'oubli ou la négligence du corps dans l'univers intellectuel à l'époque contemporaine, difficile d'imaginer d'autres postures physiques (parfois d'autres postures intellectuelles) que celles dont on a l'habitude et qu'on se laisse imposer plus ou moins

; il y a là pourtant une autre dimension d'une revitalisation possible. Il n'est pas aisé de combattre le complexe à l'égard des technologies de pointe qui iraient plus vite que

« nous

", les chercheurs et gouverneraient nombre de nos pratiques quotidiennes avec des implications cognitives de plus en plus explorées

; lorsque ce complexe s'ajoute à l'intégration d'une incompétence présumée en matière de gestion (laquelle néo-gestion mobilise ces mêmes outils informatiques pour contrôler la performance et la qualité de la recherche comme de toutes les activités humaines), alors les chercheurs sont vraiment en passe d'abandonner leur pouvoir intellectuel et politique au sein des institutions de recherche. Pour finir, il est aujourd'hui presque impossible

d'assumer les vertus de la lenteur ou, du moins, de la variabilité des rythmes, dans la gestation des recherches. Et là, nous répondons volontiers au bel appel de Gilles Clément, titulaire pour 2011-2012 de la chaire de création artistique au Collège de France, un appel exprimé lors de sa leçon inaugurale du 1

eer décembre 2011 : il invite tous les « accidentés de la vitesse

» à le rejoindre pour redessiner le jardin planétaire, son « jardin politique

» comme il l'appelle volontiers. Or le bibliothécaire n'est guère dépaysé dans cette évocation du jardin, où surgissent ça et là les mots familiers d'index et de désherbage...

Aussi n'est-il que peu surpris lorsque, de ce jardin planétaire, émergent de petites

«bibliothèques phares

", celles de Curitiba au Brésil ${ }^{13}$. Cette belle connivence invite à espérer

: le Liberlabo pourrait être demain, à son tour, sur le modèle de ces jardins réinventés, harmonieusement indexés et naturellement désherbés, notre « bibliothèque politique ». 


\section{NOTES}

1.

Le Collège de France, l'École normale supérieure, l'École nationale supérieure de chimie de Paris, l'École supérieure de physique et de chimie industrielles de la ville de Paris et l'Observatoire de Paris-Meudon sont alors les cinq membres fondateurs de la fondation de coopération scientifique Paris Sciences et Lettres.

2. Cf. quelques parcours interdisciplinaires déclinés sur le site de PSL : http://www.parissciencesetlettres.org/default/EN/all/education_fr/ physics_chemistry_and_manageme.htm

3.

Cf. schéma des gisements documentaires principaux sur le site web ayant servi à la candidature IDEX. http://www.parissciencesetlettres.org/default/EN/all/outreach_fr/ un_gisement_unique_de_sources.htm

4. IST pour information scientifique et technique.

5.

Le web de données et les outils de découverte, en constante évolution, relativisent certes cette position mais ne suffisent pas à l'infirmer.

6.

Notons que ce sont des professionnels des bibliothèques (en poste par ailleurs dans un établissement) qui ont engagé un travail bénévole de négociation pour la communauté universitaire dans le cadre du consortium Couperin, par exemple.

7. Sur toutes ces questions, nous renvoyons au numéro 26 (automne 2010) de la revueDazibao : Revue professionnelle de l'Agence régionale du livre PACA, Aix-en-Provence, depuis 2004.

8.

C'est le Liberlabo à l'état de projet qui est ici présenté dans ses aspects les plus généraux, de même que seront éclairées les conditions de sa genèse, nourrie d'une double expérience, académique - une recherche doctorale sur l'origine du service public de lecture en France - et professionnelle - une expérience de quinze ans dans une grande bibliothèque d'enseignement supérieur et de recherche, dont neuf ans de direction.

9.

Cf. le site de candidature présentait un schéma de préfiguration de quelques parcours thématiques au sein du campus

; il n'est plus disponible à ce jour.

10.

Cf. sur ces thèmes l'ouvrage de Christian Topalov consacré au séjour de Maurice Halbwachs à Chicago, et le compte rendu qu'en fait Christian Baudelot :

http://lectures.revues.org/8075

11. Thèse non publiée, intituléeLes petites bibliothèques de la République : aux origines de la lecture publique parisienne, des années 1870 aux années 1930

, sous la direction du professeur Philippe Levillain, université de Paris X-Nanterre, décembre

1998.

12.

Cf. Rosanvallon Pierre,La Société des égaux,Paris, Le Seuil, 2011

. Présentation et signature à la Librairie Compagnie, septembre 2011.

13. Gilles, Clément Jardins, paysage et génie naturel, Paris, Collège de France/Fayard, $2012\left(\mathrm{n}^{\circ} 222\right)$. 


\section{AUTEUR}

\section{LAURE LÉVEILLÉ}

Maître de conférences en histoire, actuellement chargée d'une mission scientifique au service des Archives du Collège de France.

D’octobre 2010 à décembre 2012, Laure Léveillé a coordonné les 51 bibliothèques autour du projet documentaire commun des partenaires de PSL, après en avoir imaginé les grandes lignes prospectives, dès le printemps 2010, à la demande de la Direction de l'ENS ; elle dirigeait alors le réseau des bibliothèques de l'ENS depuis 2001. Depuis décembre 2012, c'est Hélène Chaudoreille, Conservateur général, qui conduit les opérations « Ressources et Savoirs » au sein de la Fondation PSL. 